\title{
ON MULTIPLIER TRANSFORMATIONS
}

\author{
K. R. UNNI AND P. K. GeETHA*
}

(Received May 9, 1970 : Revised July 13, 1970)

1. Introduction. Let $\lambda$ be a real number such that $-\frac{1}{2}<\lambda<\frac{1}{2}$. Let $T$ denote the set of real numbers modulo one and $Z$ the additive group of integers. For $1 \leqq p<\infty$, we denote by $l^{p, \lambda}(z)$ the vector space of complex-valued functions $f$ defined on $Z$ such that

$$
N_{p, \lambda}[f]=\left\{\sum_{n \in Z}|f(n)|^{p}(|n|+1)^{p \lambda}\right\}^{1 / p}<\infty,
$$

while $L^{p, \lambda}(T)$ denotes the space of those complex-valued functions $f$ defined on $T$ for which

$$
\|f\|_{p, \lambda}=\left(\int_{T}\left|f(\theta) \theta^{\lambda}\right|^{p} d \theta\right)^{1 / p}<\infty .
$$

If $f \in l^{2,0}(Z)$, its Fourier transform

$$
f^{\wedge}(\theta)=\sum_{n \in Z} f(n) e^{2 \pi i n \theta}, \quad \theta \in T
$$

exists as a limit in the mean, of order 2 , of the partial sums of the series on the right, and the inversion formula

$$
f(n)=\int_{T} f^{\wedge}(\theta) e^{-2 \pi i n \theta} d \theta
$$

is valid. Let $h^{\wedge}$ be a bounded measurable function defined on $T$. Set

$$
H f(n)=\int_{T} f^{\wedge}(\theta) h^{\wedge}(\theta) e^{-2 \pi i n \theta} d \theta
$$

* Junior Research Fellow, C.S. I. R., Government of India. 
for $n \in Z, f \in l^{2,0}(Z)$. Such a transformation $H$, determined by $h^{\wedge}$, is called a multiplier transformation. If

$$
N_{p, \lambda}[H]=1 \text {. u. b. }\left\{N_{p, \lambda}[H f] / N_{p, \lambda}[f], f \in l^{2,0}(Z) \cap l^{p, \lambda}(Z), f \neq 0\right\}
$$

is finite, then $H$ has a unique extension, as a bounded linear transformation of $l^{p, \lambda}(Z)$ into itself, with norm $N_{p, \lambda}[H]$, since $l^{2,0}(Z) \cap l^{p, \lambda}(Z)$ is dense in $l^{p, \lambda}(Z)$.

Similarly for $f \in L^{2,0}(T)$, we set

$$
f^{\wedge}(n)=\int_{T} f(\theta) e^{-2 \pi i n \theta} d \theta
$$

Let $h^{\wedge}$ be a bounded function defined on $Z$. Then the multiplier transformation $H$, associated with $h^{\wedge}$, is defined by

$$
H f(\theta)=\sum_{n \in Z} h^{\wedge}(n) f^{\wedge}(n) e^{2 \pi i n \theta} .
$$

If

$$
\|H\|_{p, \lambda}=1 \text {. u. b. }\left\{\|H f\|_{p, \lambda} /\|f\|_{p, \lambda}, f \in L^{2,0}(T) \cap L^{p, \lambda}(T), f \neq 0\right\},
$$

is finite, then $H$ has a unique extension as a bounded linear transformation of $L^{p, \lambda}(T)$ into itself.

An important problem in this connection is to find sufficient conditions on the multiplier function $h^{\wedge}$ so that the multiplier transformation $H$ associated with $h^{\wedge}$ is bounded. In [4] Hirschman has investigated this problem when $\lambda=0$. In [6] he considered the problem for $l^{2, \lambda}(Z)$ and obtained the following result in terms of bounded $\beta$-variation of a function.

THEOREM A. Let $h^{\wedge}$ be defined on $T$ and let $H$ be the corresponding multiplier transformation. If $V_{\beta}\left[h^{\wedge}\right]$ is finite $(\beta>2)$ then

$$
N_{2, \lambda}[H]<\infty \quad \text { if }|\lambda|<\frac{1}{\beta},
$$

where $V_{\beta}\left[h^{\wedge}\right]$ denotes the $\beta$-variation of $h^{\wedge}$.

In this paper we extend the results of Hirschman to $l^{p, \lambda}(Z)$. These results are given in section 3 . In section 2 , the result analogous to Theorem $\mathrm{A}$ is given for $L^{2, \lambda}(T)$. The authors wish to express their gratitude to Professor Igari for his useful comments, particularly for the improvement on the proof of Theorem 2.6. 
2. Multipliers on $\mathbf{L}^{2, \lambda}(\mathbf{T})$. Let $h^{\wedge}$ be a bounded function defined on $Z$ and $H$ the corresponding multiplier transformation on $L^{2, \lambda}(T)$. If $I(H)$ is the set of all indices $\lambda$ for which $\|H\|_{2, \lambda}$ is finite, then it is easy to verify that

$$
\begin{aligned}
& \text { if } \lambda_{1}, \lambda_{2} \in I(H) \text { and if } \gamma=(1-\eta) \lambda_{1}+\eta \lambda_{2}, 0<\eta<1, \\
& \text { then } \gamma \in I(H) \text { and }\|H\|_{2, \gamma} \leqq\|H\|_{2, \lambda_{1}}^{1-\eta}\|H\|_{2, \lambda_{2}}^{\eta},
\end{aligned}
$$

$$
\text { if } \lambda \in I(H) \text {, then }-\lambda \in I(H) \text {, and }\|H\|_{2, \lambda}=\|H\|_{2,-2} \text {. }
$$

The first of these results is a consequence of the Riesz-Thorin convexity theorem, see [7], while the second results from the fact that the conjugate space of $L^{2, \lambda}(T)$ is $L^{2,-\lambda}(T)$.

We shall now give two lemmas that we need.

LEMMA 2.1. If $f(\theta) \sim \sum_{n \in Z} f^{\wedge}(n) e^{2 \pi i n \theta}$, then for $0 \leqq \lambda<\frac{1}{2}$,

$$
\begin{aligned}
& \sum_{n \in Z}\left|f^{\wedge}(m+n)\right|^{2}(|n|+1)^{-2 \lambda} \leqq A^{\prime}(\lambda)\|f\|_{2, \lambda}^{2} \\
& \sum_{n \in Z}\left|f^{\wedge}(m+n)\right|^{2}(|n|+1)^{2 \lambda} \geqq A^{\prime \prime}(\lambda)\|f\|_{2,-\lambda}^{2}
\end{aligned}
$$

for all $m \in Z$ where $A^{\prime}(\lambda)$ and $A^{\prime \prime}(\lambda)$ are positive constants depending only on $\lambda$.

This can be easily deduced from Hirschman [3, p. 51].

LEMMA 2.2. If $f \in L^{2, \lambda}(T)$ and if $a_{n}=\int_{T} f(\theta) e^{-2 \pi i n \theta} d \theta$. then for $0<\lambda$ $<\frac{1}{2}$

$$
A^{\prime} \int_{T}\left|f(\theta) \theta^{\lambda}\right|^{2} d \theta \leqq \sum_{n=1}^{\infty} \sum_{m=-\infty}^{\infty}\left|a_{n+m}-a_{m}\right|^{2} n^{-1-2 \lambda} \leqq A^{\prime \prime} \int_{T}\left|f(\theta) \theta^{\lambda}\right|^{2} d \theta
$$

where $A$ and $A^{\prime \prime}$ are positive constants depending only on $\lambda$. (See Hirschman $[3$, p. 52]).

Let $\mathfrak{M}_{\lambda}$ denote the set of all bounded multiplier transformations on $L^{2, \lambda}(T)$. 
THEOREM 2.3. Suppose $0<\lambda<\frac{1}{2}$ and $H \in \mathfrak{M}_{\lambda}$. Then there exists a constant $A^{\prime}(\lambda)$ such that for any $f \in L^{2, \lambda}(T)$,

(1) $\quad \sum_{n=1}^{\infty} n^{-1-2 \lambda} \sum_{m=-\infty}^{\infty}\left|f^{\wedge}(m)\right|^{2}\left|h^{\wedge}(m+n)-h^{\wedge}(m)\right|^{2} \leqq A^{\prime}(\lambda)\|H\|_{2, \lambda}^{2}\|f\|_{2, \lambda}^{2}$.

Proof. From the relation

$$
\begin{aligned}
f^{\wedge}(m)\left[h^{\wedge}(m+n)-h^{\wedge}(m)\right]= & {\left[f^{\wedge}(m+n) h^{\wedge}(m+n)-f^{\wedge}(m) h^{\wedge}(m)\right] } \\
& +\left[f^{\wedge}(m)-f^{\wedge}(m+n)\right] h^{\wedge}(m+n)
\end{aligned}
$$

it follows that, since $\left|h^{\wedge}(m+n)\right| \leqq\|H\|_{2, \lambda}$, as can be easily verified,

$$
\begin{aligned}
\left|f^{\wedge}(m)\right|^{2}\left|h^{\wedge}(m+n)-h^{\wedge}(m)\right|^{2} \leqq & 2\left|f^{\wedge}(m+n) h^{\wedge}(m+n)-f^{\wedge}(m) h^{\wedge}(m)\right|^{2} \\
& +2\|H\|_{2, \lambda}^{2}\left|f^{\wedge}(m+n)-f^{\wedge}(m)\right|^{2} .
\end{aligned}
$$

Multiplying by $n^{-1-2 \lambda}$ and summing over $m$ and $n$, we get the desired result, using Lemma 2. 2.

THEOREM 2.4. Let $0<\lambda<\frac{1}{2}$. There exists a constant $A^{\prime \prime}(\lambda)$ such that if $h^{\wedge}$ is defined on $Z$ satisfying

$$
\left|h^{\wedge}(m)\right| \leqq C \quad m \in Z
$$

and

$$
\sum_{n=1}^{\infty} n^{-1-2 \lambda} \sum_{m=-\infty}^{\infty}\left|f^{\wedge}(m)\right|^{2}\left|h^{\wedge}(m+n)-h^{\wedge}(m)\right|^{2} \leqq C^{2} .\|f\|_{2, \lambda}^{2},
$$

for every $f \in L^{2, \lambda}(T)$, then $H \in \mathfrak{M}_{\lambda}$, and $\|H\|_{2, \lambda} \leqq A^{\prime \prime}(\lambda) C$.

ProOF. We have

$$
\begin{aligned}
f^{\wedge}(m+n) h^{\wedge}(m+n)-f^{\wedge}(m) h^{\wedge}(m)= & f^{\wedge}(m)\left[h^{\wedge}(m+n)-h^{\wedge}(m)\right] \\
& +\left[f^{\wedge}(m+n)-f^{\wedge}(m)\right] h^{\wedge}(m+n)
\end{aligned}
$$

so that 


$$
\begin{aligned}
& \left|f^{\wedge}(m+n) h^{\wedge}(m+n)-f^{\wedge}(m) h^{\wedge}(m)\right|^{2} \\
\leqq & 2\left|f^{\wedge}(m)\right|^{2} \cdot\left|h^{\wedge}(m+n)-h^{\wedge}(m)\right|^{2}+2 C^{2}\left|f^{\wedge}(m+n)-f^{\wedge}(m)\right|^{2} .
\end{aligned}
$$

Multiplying by $n^{-1-2 \lambda}$ and summing over $m$ and $n$, the desired result follows by virtue of Lemma 2.2.

THEOREMS 2.3 and 2.4 correspond to the results of Devinatz and Hirschman [1, Lemmas $3 \mathrm{~d}, 3 \mathrm{e}]$.

Before we prove our main result in this section, we need the following definition.

DEFINITION 2.5. If $g^{\wedge}$ is a function defined on $Z$, then we define

$$
V_{\beta}\left[g^{\wedge}\right]=1 \text { u. b. }\left\{\sum_{k=0}^{N-1}\left|g^{\wedge}\left(n_{k+1}\right)-g^{\wedge}\left(n_{k}\right)\right|^{\beta}\right\}^{1 / \beta},
$$

the least upper bound being taken over all sets of integers $n_{0}<n_{1}<n_{2}<\cdots<n_{N^{*}}$ and it is called the $\beta$-variation of $g^{\wedge}$.

First we prove a result analogous to the lemma of Hirschman [6].

THEOREM 2.6. Suppose that $0<\lambda<\frac{1}{2}$. Let $h^{\wedge}$ be of bounded 1-variation on $Z$. Then, if $H$ is the corresponding multiplier transformation, we have

$$
\|H\|_{2, \lambda}^{2} \leqq B(\lambda)\left\{\left\|h^{\wedge}\right\|_{\infty}^{2}+\left\|h^{\wedge}\right\|_{\infty} V_{1}\left[h^{\wedge}\right]\right\}
$$

where $B(\lambda)$ is a finite constant depending only on $\lambda$ and

$$
\left\|h^{\wedge}\right\|_{\infty}=\sup _{n \in Z}\left|h^{\wedge}(n)\right|
$$

ProOF. By virtue of theorem 2.4, we need only to estimate the quantity $M=\sum_{n=1}^{\infty} n^{-1-2 \lambda} \sum_{m \in Z}\left|f^{\wedge}(m)\right|^{2}\left|h^{\wedge}(m+n)-h^{\wedge}(m)\right|^{2}$.

Now

$$
M \leqq 2\left\|h^{\wedge}\right\|_{\infty} \sum_{n=1}^{\infty} n^{-1-2 \lambda} \sum_{m=-\infty}^{\infty}\left|f^{\wedge}(m)\right|^{2} \sum_{k=1}^{n}\left|h^{\wedge}(m+k)-h^{\wedge}(m+k-1)\right|
$$




$$
\begin{aligned}
& =2\left\|h^{\wedge}\right\|_{\infty} \sum_{n=1}^{\infty} n^{-1-2 \lambda} \sum_{k=1}^{n} \sum_{m=-\infty}^{\infty}\left|f^{\wedge}(m-k)\right|^{2}\left|h^{\wedge}(m)-h^{\wedge}(m-1)\right| \\
& =2\left\|h^{\wedge}\right\|_{\infty} \sum_{m=-\infty}^{\infty}\left|h^{\wedge}(m)-h^{\wedge}(m-1)\right| \sum_{k=1}^{\infty}\left|f^{\wedge}(m-k)\right|^{2} \sum_{n=k}^{\infty} n^{-1-2 \lambda} \\
& \leqq \frac{1}{\lambda}\left\|h^{\wedge}\right\|_{\infty} \sum_{m=-\infty}^{\infty}\left|h^{\wedge}(m)-h^{\wedge}(m-1)\right| \sum_{k=1}^{\infty}\left|f^{\wedge}(m-k)\right|^{2} k^{-2 \lambda} \\
& \leqq C(\lambda)\left\|h^{\wedge}\right\|_{\infty} V_{1}\left[h^{\wedge}\right]\|f\|_{2, \lambda}^{2}
\end{aligned}
$$

using Lemma 2.1.

LEMMA 2.7. Let $h^{\wedge}$ be a real valued function defined on $Z$. For each $\beta>1$, there exists a constant $C(\beta)$, depending only on $\beta$, such that for each $h^{\wedge}$ for which $V_{\beta}\left[h^{\wedge}\right]<\infty$ and $\varepsilon>0$, there exists $h_{\varepsilon}^{\wedge}$ with the following properties :

(a) $\left\|h^{\wedge}-h_{\varepsilon}^{\wedge}\right\|_{\infty} \leqq \varepsilon$,

(b) $V_{1}\left[h^{\wedge}\right] \leqq C(\beta) V_{\beta}\left[h^{\wedge}\right]^{\beta} \varepsilon^{1-\beta}$,

where $\|\cdot\|_{\infty}$ is defined as in Theorem 2.6.

This lemma corresponds to Lemma 3 of Hirschman in [6] and can be proved by the arguments used in [4].

We now come to the main result in this section and it is the analogue of Theorem A stated in the introduction.

THEOREM 2.8. Let $h^{\wedge}$ be defined on $Z$ and let $H$ be the corresponding multiplier transformation on $L^{2, \lambda}(T)$. If $V_{\beta}\left[h^{\wedge}\right]$ is finite, where $\beta>2$, then

$$
\|H\|_{2, \lambda}<\infty \quad \text { if }|\lambda|<\frac{1}{\beta} .
$$

PROOF. First we obtain a sequence of functions $g^{\wedge}{ }_{m}$ such that

$$
h^{\wedge}=\lim _{m \rightarrow \infty} g^{\wedge}
$$

pointwise on $Z$. This construction is given by Hirschman [4] (see also Edwards [2, Vol.2, p. 270]). We shall not give the details here. Assuming without loss 
of generality that $h^{\wedge}(0)=0$, a real valued function $h^{*}$ on the entire real line is obtained by interpolating linearly between successive values of $h^{\wedge}(n)$ so that $\left.h^{*}(x)\right|_{x=n}=h^{\wedge}(n)$. Then for each positive integer $m$, a function $g^{\wedge}{ }_{m}$ is constructed satisfying

$$
V_{1}\left[g_{m}^{\wedge}\right] \leqq 2^{(\beta-1) m} V_{\beta}\left[h^{\wedge}\right]^{\beta}
$$

and

$$
\left\|h^{\wedge}-g_{m}^{\wedge}\right\|_{\infty} \leqq 2^{-m}
$$

Furthermore

$$
V_{\beta}\left[g^{\wedge}{ }_{m}\right] \leqq V_{\beta}\left[h^{\wedge}\right]
$$

The proof of our theorem is completed following the arguments of Hirschman [6]. Define a sequence of functions $\left\{h^{\wedge}\right\}_{m=1}^{\infty}$ on $Z$ as follows :

$$
\begin{gathered}
h^{\wedge}{ }_{1}(n)=g^{\wedge}{ }_{1}(n) \\
h^{\wedge}{ }_{m}(n)=g^{\wedge}{ }_{m}(n)-g^{\wedge}{ }_{m-1}(n) .
\end{gathered}
$$

Then

$$
h^{\wedge}(n)=\sum_{m=1}^{\infty} h_{m}^{\wedge}(n)
$$

and

$$
\begin{gathered}
V_{1}\left[h^{\wedge}{ }_{m}\right] \leqq C .2^{(\beta-1) m} V_{\beta}\left[h^{\wedge}\right]^{\beta} \\
\left\|h^{\wedge}{ }_{m}\right\|_{\infty} \leqq C 2^{-m}
\end{gathered}
$$

If $H_{m}$ is the multiplier transformation associated with $h^{\wedge}{ }_{m}$, then

$$
\|H\|_{2, \lambda} \leqq \sum_{m=1}^{\infty}\left\|H_{m}\right\|_{2, \lambda}
$$

Choose $\alpha, \lambda<\alpha<\frac{1}{2}$. By Theorem 2.6,

$$
\left\|H_{m}\right\|_{2, \alpha}=O\left[\left(2^{-m}\right)^{2}+2^{-m} \cdot 2^{m(\beta-1)}\right]^{1 / 2}=\mathrm{O}\left(2^{m(\beta / 2-1)}\right) .
$$


On the other hand, by Parseval's equality

$$
\left\|H_{m}\right\|_{2,0}=\left\|h_{m}^{\wedge}\right\|_{\infty}=O\left(2^{-m}\right) \text {. }
$$

Putting $\lambda=(1-\theta) 0+\theta \alpha, 0<\theta<1$, we obtain by the Riesz-Thorin convexity theorem,

$$
\left\|H_{m}\right\|_{2, \lambda}=O\left(2^{m(-1+\beta \lambda / 2 \alpha)}\right)
$$

The series $\sum_{m=1}^{\infty}\left\|H_{m}\right\|_{2, \lambda}$ is convergent if $\lambda<\frac{2 \alpha}{\beta}$. Since $\alpha$ is arbitrary such that $\lambda<\alpha<\frac{1}{2}$, it is always possible to choose $\alpha$ so that $\lambda<\frac{2 \alpha}{\beta}$ if $0<\lambda<\frac{1}{\beta}$. Thus we have proved our theorem if $0<\lambda<\frac{1}{\beta}$. The case when $\lambda=0$ being trivial, the theorem follows from the duality argument given at the beginning of this section.

3. Multipliers on $\boldsymbol{l}^{p, \lambda}(\boldsymbol{Z})$. We shall now consider the problem for $l^{p, \lambda}(Z)$ and obtain some results similar to those given by Hirschman [4] for the case $\lambda=0$. Let $f \in l^{2,0}(Z)$. If

$$
h(k)=\int_{T} h^{\wedge}(\theta) e^{-2 \pi i k^{\theta}} d \theta \quad k \in Z
$$

then

$$
H f(n)=\sum_{k \in Z} f(n-k) h(k)
$$

The series on the right converges absolutely for each $n$, by Parseval's relation. If $1 / p+1 / q=1$, then it is easy to verify that if $H$ is a multiplier transformation on $l^{p, \lambda}(Z)$ then $H$ is also a multiplier transformation on $l^{q,-\lambda}(Z)$ associated with the same $h^{\wedge}$ and $N_{p, \lambda}[H]=N_{q,-\lambda}[H]$.

THEOREM 3.1. If
(a) $\left|h^{\wedge}(\theta)\right| \leqq A$
$\theta \in T$
(b) $\left|h^{\wedge}(\theta)-h^{\wedge}(\theta+t)\right| \leqq A|t|^{\alpha}$
$1 / 2<\alpha \leqq 1$

then $H$ is a bounded linear transformation of $l^{p, \lambda}(Z)$ into itself where $1<p$ 
$<\infty$ and $\frac{1}{2}-\alpha<\lambda<\alpha-\frac{1}{2}$.

PROOF. Let

$$
s_{k}^{\wedge}(\theta)=\sum_{|n| \leq 2^{k}} h(n) e^{2 \pi i n \theta}
$$

be the partial sum of order $2^{k}$ of the Fourier series for $h^{\wedge}$. Given $\varepsilon>0$, it is easily seen that

$$
\left\|{s^{\wedge}}_{k}-h^{\wedge}\right\|_{\infty} \leqq A C(\alpha, \varepsilon) 2^{-k(\alpha-\varepsilon)}
$$

(Zygmund [7, p.61], Hirschman [4, p. 223]) so that if

$$
h^{\wedge}{ }_{k}={s^{\wedge}}_{k}-{s^{\wedge}}_{k-1}
$$

then

$$
\left\|h^{\wedge}{ }_{k}\right\|_{\infty} \leqq A C(\alpha, \varepsilon) 2^{-k(\alpha-\varepsilon)}
$$

where $\|\cdot\|_{\infty}$ is on $T$. Let $H_{k}$ be the multiplier transformation associated with $h^{\wedge}{ }_{k}$. Then

$$
H_{k} f(n)=\int_{T} f^{\wedge}(\theta) h^{\wedge}{ }_{k}(\theta) e^{-2 \pi i n \theta} d \theta=\sum_{j \in Z_{k}} f(n-j) h(j)
$$

where $Z_{k}=\left\{n \in Z, 2^{k-1}<|n| \leqq 2^{k}\right\}$ and

$$
N_{r, \lambda}\left[H_{k}\right] \leqq\left\{\sum_{j \in Z_{k}}|h(j)|^{r}(1+|j|)^{r \lambda}\right\}^{1 / r} \quad r=1,2 .
$$

Using the relation

$$
\sum_{j \in Z_{k}}|h(j)| \leqq A C(\alpha, \varepsilon) 2^{k(1 / 2-\alpha+\varepsilon)}
$$

it easily follows that

$$
N_{1, \lambda}\left[H_{k}\right] \leqq A C(\alpha, \varepsilon) 2^{k(1 / 2-\alpha+\varepsilon+|\lambda|)}
$$

From (4) and (5) using Schwartz inequality and Parseval's relation, it follows 
that

$$
N_{2, \lambda}\left[H_{k}\right] \leqq A C(\alpha, \varepsilon) 2^{k(1 / 2+|\lambda|+\varepsilon-\alpha)}
$$

Suppose $1<p \leqq 2$. Putting $\frac{1}{p}=\frac{1-\omega}{1}+\frac{\omega}{2}, 0<\omega<1$, we obtain from $(6)$ and ( 7 ) by virtue of Riesz-Thorin convexity theorem

$$
N_{p, \lambda}\left[H_{k}\right] \leqq A C(\alpha, \varepsilon) 2^{k(1 / 2+|\lambda|+\varepsilon-\alpha)}
$$

If $|\lambda|<\alpha-\frac{1}{2}$, we can choose $\varepsilon$ so small that

$$
\sum_{k=0}^{\infty} N_{p, \lambda}\left[H_{k}\right]<\infty
$$

Further since $h^{\wedge}(\theta)=\sum_{k=0}^{\infty} h^{\wedge}{ }_{k}(\theta)$, the convergence being uniform in $\theta$ it is easy to see that $H f(n)=\sum_{k=0}^{\infty} H_{k} f(n)$ and $N_{p, \lambda}[H] \leqq \sum_{k=0}^{\infty} N_{p, \lambda}\left[H_{k}\right]<\infty$. The regular conjugacy argument gives the result for $2 \leqq p<\infty$.

Now we state two results of Devinatz and Hirschman [1] as lemmas.

LEMMA 3.2. If $0<\lambda<1 / 2$, then there exist positive constants $A_{1}(\lambda)$ and $A_{2}(\lambda)$ depending only on $\lambda$ such that

$$
\left(N_{2, \lambda}[f]\right)^{2}-|f(0)|^{2} \leqq A_{1}(\lambda) \int_{0}^{1} \int_{0}^{1}\left\{\left|f^{\wedge}(\theta)-f^{\wedge}(\phi)\right|^{2}(\sin \pi|\theta-\phi|)^{-1-2 \lambda}\right\} d \theta d \phi
$$

and

$$
\left(N_{2, \lambda}[f]\right)^{2}-|f(0)|^{2} \geqq A_{2}(\lambda) \int_{0}^{1} \int_{0}^{1}\left\{\left|f^{\wedge}(\theta)-f^{\wedge}(\phi)\right|^{2}(\sin \pi|\theta-\phi|)^{-1-2 \lambda}\right\} d \theta d \phi .
$$

LEMMA 3.3. Let $0<\lambda<\frac{1}{2}$. There exists a constant $A^{\prime \prime}(\lambda)$ such that if $h^{\wedge}$ is a measurable function on T satisfying $h(0)=0$,

$$
\left\|h^{\wedge}\right\|_{\infty} \leqq C
$$


and

$$
\int_{T}\left|f^{\wedge}(\theta)\right|^{2} d \theta \int_{T}\left|h^{\wedge}(\theta)-h^{\wedge}(\phi)\right|^{2}(\sin \pi|\theta-\phi|)^{-1-2 \lambda} d \phi \leqq C^{2}\left(N_{2, \lambda}[f]\right)^{2}
$$

for every $f \in l^{2, \lambda}(Z)$, then $N_{2, \lambda}[H] \leqq A^{\prime \prime}(\lambda) C$.

We now prove and

THEOREM 3.4. Suppose $h^{\wedge}$ satisfies the condition (a) of Theorem 3.1

$$
\left|h^{\wedge}(\theta)-h^{\wedge}(\theta+t)\right| \leqq B|t|^{\alpha} \quad 0<\alpha \leqq 1
$$

Then there exists a constant $C$ such that

$$
\int_{T}\left|f^{\wedge}(\theta)\right|^{2} d \theta \int_{T}\left|h^{\wedge}(\theta)-h^{\wedge}(\phi)\right|^{2}(\sin \pi|\theta-\phi|)^{-1-2 \lambda} d \theta d \phi \leqq C A B\left(N_{2, \lambda}[f]\right)^{2}
$$

where $0<\lambda<\alpha / 2$.

PROOF. We consider the quantity

$$
\begin{aligned}
M & =\int_{T}\left|f^{\wedge}(\theta)\right|^{2} \int_{T}\left|h^{\wedge}(\theta)-h^{\wedge}(\phi)\right|^{2}(\sin \pi|\theta-\phi|)^{-1-2 \lambda} d \theta d \phi \\
& \leqq 2\left\|h^{\wedge}\right\|_{\infty} \int_{T}\left|f^{\wedge}(\theta)\right|^{2} \int_{T}\left|h^{\wedge}(\theta)-h^{\wedge}(\phi)\right|(\sin \pi|\theta-\phi|)^{-1-2 \lambda} d \theta d \phi .
\end{aligned}
$$

It is easy to establish that there exists a constant $C$ which depends on $\lambda$ and $\alpha$ such that

$$
\int_{T}\left|h^{\wedge}(\theta)-h^{\wedge}(\phi)\right|(\sin \pi|\theta-\phi|)^{-1-2 \lambda} d \phi \leqq C
$$

Thus

$$
M \leqq C\left\|h^{\wedge}\right\|_{\infty} \int_{T}\left|f^{\wedge}(\theta)\right|^{2} d \theta \leqq C\left\|h^{\wedge}\right\|_{\infty} \int_{0}^{1}\left|f^{\wedge}(\theta)\right|^{2} \theta^{-2 \lambda} d \theta
$$


when $\lambda>0$. Now applying Lemma 2.1 we obtain

$$
M \leqq C\left\|h^{\wedge}\right\|_{\infty}\left(N_{2, \lambda}[f]\right)^{2}
$$

where $C$ is a constant depending on $\lambda$ and $\alpha$ only.

THEOREM 3.5. Suppose $h^{\wedge}$ satisfies the conditions of theorem 3.4. Then if $0<\lambda<\frac{\alpha}{2}$, there exists a constant $C$ which depends on $\alpha$ and $\lambda$ such that if $H$ is the associated multiplier transformation such that $h(0)=0$, then

$$
\left(N_{2, \lambda}[H]\right)^{2} \leqq C A B
$$

PROOF. An application of Lemmas 3.2 and 3.3 together with Theorem 3.4 gives the result.

THEOREM 3.6. Suppose $h^{\wedge}$ satisfies the condition of Theorem 3. 1. Then $H$ is a bounded linear transformation of $l^{p, \lambda}(Z)$ into itself, where ${ }_{2}^{\alpha}>|\lambda|>\alpha-\frac{1}{2}$ and

$$
\frac{2(1-\alpha+2|\lambda|)}{1+2|\lambda|}<p<\frac{2(1-\alpha+2|\lambda|)}{1+2|\lambda|-2 \alpha}
$$

Proof. Suppose $s^{\wedge}{ }_{k}$ is defined in the proof of Theorem 3.1 and $H_{k}$ the multiplier transformation defined there. Then since

$$
\left\|{s^{\wedge}}_{k}\right\|_{\infty} \leqq A C(\alpha, \varepsilon) 2^{-k(\alpha-\varepsilon)}
$$

and, as can be easily verified,

$$
\left|h^{\wedge}{ }_{k}(\theta)-h^{\wedge}{ }_{k}(\theta+t)\right| \leqq A C(\alpha, \varepsilon) 2^{* k}|t|^{\alpha}
$$

we have by virtue of Theorem 3.5

$$
N_{2, \lambda}^{2}\left[H_{k}\right] \leqq A 2^{-k(\alpha-\varepsilon)}
$$

which implies that

$$
N_{2, \lambda}\left[H_{k}\right] \leqq A \cdot 2^{-k / 2(\alpha-\varepsilon)}
$$


Now suppose $\frac{2(1-\alpha+2|\lambda|)}{1+2|\lambda|}<p \leqq 2$. Then if $1 / p=(1-\omega) / 1+\omega / 2$ we have $\omega>$ $\frac{1-2 \alpha+2|\lambda|}{1-\alpha+2|\lambda|}$. By the Riesz-Thorin convexity theorem (this is possible since $0<\omega<1$ under the condition that $|\lambda|>\alpha-1 / 2)$ we obtain from (6) and (8)

$$
N_{p, \lambda}\left[H_{k}\right] \leqq A 2^{k[(1 / 2-\alpha+|\lambda|+\varepsilon)(1-\omega)-\omega(\alpha-\varepsilon) / 2]}
$$

Now under the above condition on $\omega$, it is possible to choose $\varepsilon$ small enough such that the quantity in the exponent of the right hand side of $(9)$ is negative and we obtain the result for $\frac{2(1-\alpha+2|\lambda|)}{1+2|\lambda|}<p \leqq 2$. The result for $2 \leqq p<\frac{2(1-\alpha+2|\lambda|)}{1+2|\lambda|-2 \alpha}$ follows by the conjugacy argument.

In theorems 3.1 and 3.6 we have assumed that $\alpha>\frac{1}{2}$. We have not asserted that they are the best possible. There are multiplier transformations for some $p$ and $\lambda$ even if $\alpha<\frac{1}{2}$ as can be seen from the following result.

THEOREM 3.7. If $h^{\wedge}$ satisfies conditions of Theorem 3.4, then $H$ is a bounded linear transformation of $l^{p, \lambda}(Z)$ into itself if $\frac{2}{1+2(\alpha-\lambda)}<p<2$ and $\lambda$ is a nonnegative number such that $\alpha>\lambda>\alpha-\frac{1}{2}$.

PROOF. With the notations as in the proof of Theorem 3.1 we have

$$
N_{2,0}\left[H_{k}\right] \leqq A C(\alpha, \varepsilon) 2^{-k(\alpha-\varepsilon)}
$$

Let $\gamma=(2-p) / p$. Then $1 / p=(1-\gamma) / 2+\gamma / 1$ and let $\lambda=(1-\gamma) 0+\gamma \eta$. Applying Riesz Thorin theorem to $(10)$ and to

$$
N_{1, \eta}\left[H_{k}\right] \leqq A C(\alpha, \varepsilon) 2^{k(1 / 2-\alpha+\eta+\varepsilon)}
$$

we obtain Theorem 3.7. 
REMARK. If $\alpha<1 / 2$, then $\lambda>\alpha-1 / 2$ is satisfied by any nonnegative $\lambda$. In particular when $\lambda=0$ the range for $p$ reduces to $2 /(1+2 \alpha)<p<2$ and this is the result given by Hirschman [4, Th. 2a].

\section{REFERENCES}

[1] A. Devinatz and I. I. Hirschman, JR., Multiplier transformations on $l^{2, \alpha}$, Ann. of Math., 69(1959), 575-587.

[2] R. E. EDWARDS, Fourier series, Vols. I, II, Holt, Rinehart and Winston Inc., New York, 1967.

[3] I. I. HiRschman, The decomposition of Walsh and Fourier series, Mem. Amer. Math. Soc., No. 15(1955).

[4] I. I. HIRSCHMAN, On multiplier transformations, Duke Math. J., 26(1959), 221-242.

[5] I. I. HiRschmaN, Multiplier transformations II, Duke Math. J., 28(1961), 45-56.

[6] I. I. Hirschman, Multiplier transformations III, Proc. Amer. Math. Soc., 13(1962), 851-857.

[7] A. ZYGMUND, Trigonometric series, 1935.

\section{MATSCIENCE}

THE INSTITUTE OF MATHEMATICAL SCIENCES

ADYAR, MADRAS, INDIA 